\title{
The LIFEspan model of transitional rehabilitative care for youth with disabilities: Healthcare professionals' perspectives on service delivery
}

\author{
Yani Hamdani ${ }^{\mathrm{a}, \mathrm{b}, *}$, Meghann Proulx ${ }^{\mathrm{c}}$, Shauna Kingsnorth ${ }^{\mathrm{b}, \mathrm{c}, \mathrm{d}}$, Sally Lindsay ${ }^{\mathrm{b}, \mathrm{c}, \mathrm{e}}$, Joanne Maxwell ${ }^{\mathrm{b}, \mathrm{d}, \mathrm{f}}$, \\ Angela Colantonio ${ }^{\mathrm{a}, \mathrm{b}, \mathrm{e}, \mathrm{f}}$, Colin Macarthur ${ }^{\mathrm{g}}$ and Mark Bayley ${ }^{\mathrm{f}, \mathrm{h}}$ \\ ${ }^{a}$ Dalla Lana School of Public Health, University of Toronto, Toronto, Canada \\ ${ }^{\mathrm{b}}$ Department of Occupational Science and Occupational Therapy, University of Toronto, Toronto, Canada \\ ${ }^{\mathrm{c}}$ Bloorview Research Institute, Toronto, Canada \\ ${ }^{\mathrm{d}}$ Holland Bloorview Kids Rehabilitation Hospital, Toronto, Canada

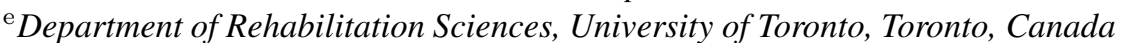 \\ ${ }^{\mathrm{f}}$ Toronto Rehab Institute, University Health Network, Toronto, Canada \\ ${ }^{\mathrm{g}}$ Hospital for Sick Children, Toronto, Canada \\ ${ }^{\mathrm{h}}$ Faculty of Medicine, University of Toronto, Toronto, Canada
}

\begin{abstract}
.
PURPOSE: LIFEspan is a service delivery model of continuous coordinated care developed and implemented by a crossorganization partnership between a pediatric and an adult rehabilitation hospital. Previous work explored enablers and barriers to establishing the partnership service. This paper examines healthcare professionals' (HCPs') experiences of 'real world' service delivery aimed at supporting transitional rehabilitative care for youth with disabilities.

METHODS: This qualitative study - part of an ongoing mixed method longitudinal study - elicited HCPs' perspectives on their experiences of LIFEspan service delivery through in-depth interviews. Data were categorized into themes of service delivery activities, then interpreted from the lens of a service integration/coordination framework.

RESULTS: Five main service delivery themes were identified: 1) addressing youth's transition readiness and capacities; 2) shifting responsibility for healthcare management from parents to youth; 3) determining services based on organizational resources; 4) linking between pediatric and adult rehabilitation services; and, 5) linking with multi-sector services.

CONCLUSIONS: LIFEspan contributed to service delivery activities that coordinated care for youth and families and integrated inter-hospital services. However, gaps in service integration with primary care, education, social, and community services limited coordinated care to the rehabilitation sector. Recommendations are made to enhance service delivery using a systems/sector-based approach.
\end{abstract}

Keywords: Transition, childhood disability, young adults, service delivery, coordinated care, continuity of care

\footnotetext{
*Corresponding author: Yani Hamdani, Social and Behavioral Health Sciences, CIHR Public Health Policy, Dalla Lana School of Public Health, University of Toronto, 155 College Street, $6^{\text {th }}$ Floor,
}

Toronto, ON, M5T 3M7 Canada. Tel.: +1 647274 9264; E-mail: y.hamdani@utoronto.ca. 


\section{Introduction}

Advances in social and medical care have allowed young people with childhood-onset physical disabilities, such as cerebral palsy $(\mathrm{CP})$ and acquired brain injury (ABI), to live longer [1,2]. In Ontario, Canada, children have access to publically funded pediatric rehabilitation services until they are 19 years old. After this age, provision of continuous care is constrained by poor linkages between pediatric and adult services coupled with a healthcare system not yet designed to meet lifelong rehabilitation needs of young adults with childhood-onset physical disabilities [3-5]. As a consequence, these young adults lack access to comprehensive and coordinated rehabilitation services, as well as age and developmentally appropriate health and community services. The increased risk and realization of poor outcomes for their health [6-10] and related domains, such as education, employment, and social relationships, have been well documented [1,11-15].

In reaction to these circumstances, two rehabilitation hospitals partnered to develop the LIFEspan (Living Independently and Fully Engaged) model - a service delivery model designed to coordinate transfer of healthcare by formally linking pediatric and adult rehabilitation services. Model development was guided by key best practice principles and recommendations reported in the literature $[16,17]$. Little is known about how these best practices influence service delivery and continuity of coordinated care across rehabilitation services. Examining the actual practical experiences of implementing the LIFEspan model can provide valuable insight into the scope and extent of service delivery that is required to improve rehabilitative care transitions for youth with disabilities. Thus, the purpose of this paper is to examine 'real world' service delivery of coordinated transitional care from the perspectives of healthcare professionals (HCPs) involved in LIFEspan model implementation.

\subsection{The LIFEspan model}

The LIFEspan model was developed to maintain continuity of coordinated care by bridging three healthcare provision phases: pediatrics, transfer, and adult services. Fully operational since 2008, LIFEspan currently serves youth with $\mathrm{CP}$ and $\mathrm{ABI}$ and their families. A hallmark feature of the model is the formal organizational linkage reflected in a multi-profession clinical team with cross-appointments at the pediatric and the adult hospital. LIFEspan provides a two-year transition preparation followed by a coordinated transfer to the adult hospital for ongoing care. Details describing guiding principles, core components, and the funding structure have previously been described by Kingsnorth et al. [16]. An overview of the LIFEspan model and core health disciplines is provided in Fig. 1.

\subsection{Service delivery activities}

For the purpose of this study, "service delivery activities" refer to any interventions or activities aimed at changing or improving transitional rehabilitative care, be it direct client- or family-based interventions or procedural/policy interventions to improve transfer processes between organizations and service coordination. Teams at both hospitals provide youth with transitional rehabilitative care-coordination (see Fig. 1) emphasizing the five domains of the "Growing $U p$ Ready" framework [16]: Parenting/advocacy; Social; Self-care; Education; and, Medical. Service delivery activities aligned with guiding transition principles include, but are not limited to:

1. Planning and coordinating the transfer of rehabilitation services with youth and their families through established processes;

2. Linking youth and families to service and supports within the rehabilitation sector as well as broader healthcare and community service sectors (e.g., employment, post-secondary education, personal care and income services and supports);

3. Developing skills and knowledge for self-advocacy and self-management in youth;

4. Establishing processes for guardianship and responsibility for healthcare with parents; and,

5. Performing client assessments based on individualized goals [16].

\subsection{The LETS study}

This unique collaborative cross-hospital partnership holds promising and significant implications as a model to address health service continuity across the pediatric/adult divide. The current study represents one component of an ongoing study (LETS: Longitudinal Evaluation of Transition Services) aimed at evaluating the impact of coordinated healthcare transfer through LIFEspan model implementation [17]. Prior work examined the perspectives of a broad group of clinical and managerial stakeholders during model development and early implementation. Findings high- 

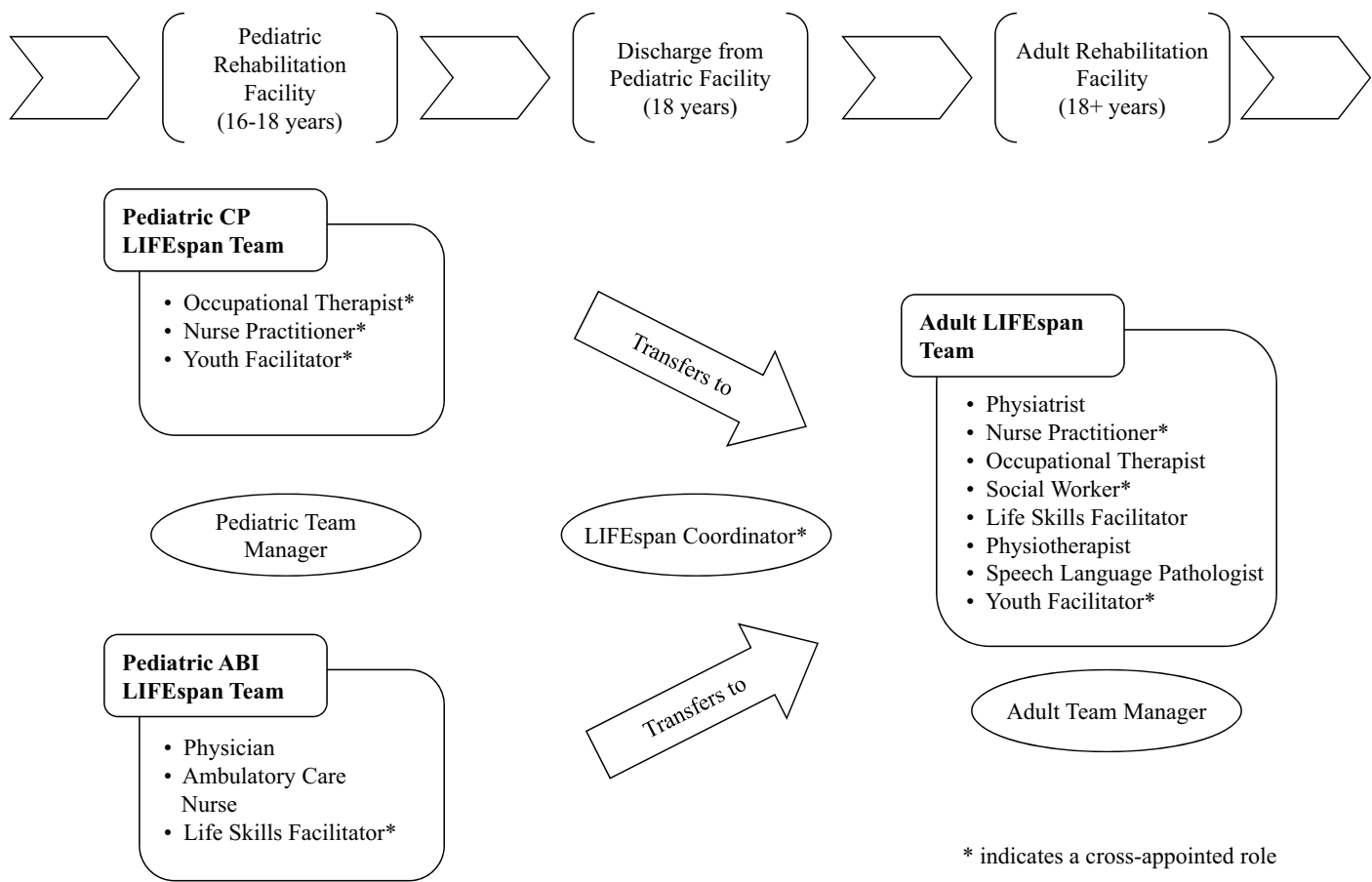

Fig. 1. The LIFEspan model.

lighted the importance of leadership, equitable commitment of resources from each organization, compatibility, and effective communication to overcome varied logistical implementation barriers (e.g., policies, role delineation and information transfer) in establishing this cross-hospital model [16].

The current study extends this work by examining the experiences of LIFEspan staff in 'real world' service delivery three years after model implementation and provision of transitional rehabilitative care to over 280 people. The specific aims are: (i) to identify and understand key LIFEspan service delivery activities related to continuity of care and, (ii) to identify successes and challenges encountered in service delivery.

\section{Design and methods}

This descriptive qualitative study involved semistructured, in-depth interviews with HCPs involved in the delivery of LIFEspan services. This data collection method gathers opinions about a particular topic using open-ended questions [18,19]. Interviews began by asking about staff experiences with service delivery compared to findings from the previous study [16] and progressed to asking about perspectives on operationalizing the model and the impact on coordi- nated transitional care. Further questions probed for successes, challenges, and suggestions for improving coordinated care at both the pediatric and adult sites based on their first-hand experiences. Examples of questions are provided in Table 1. Ethical approval was obtained from research ethics boards of both rehabilitation hospitals. The LETS study is registered as a clinical trial [17].

\subsection{Interview participants}

Fourteen HCPs in total were identified as working full- or part-time within the LIFEspan program at either the pediatric or adult rehabilitation hospital. All 14 staff members were invited to participate by letter and all agreed to participate; informed consent was obtained verbally and in writing by a research assistant (RA). The sample included 3 managerial and 11 clinical staff, with 7 staff having cross-appointments and representing a range of professional disciplines (see Fig. 1); 93\% had worked within the program since its launch in 2008. Audio-recorded interviews were arranged at the convenience of participants and conducted by an RA trained in qualitative methods in person (12 HCPs) and by phone (2 HCPs). The semistructured interviews lasted between 22 and 60 minutes, with an average length of 37 minutes. 
Table 1

Interview guide for LIFEspan healthcare professionals

1. During the first set of interviews, key enablers and barriers to the LIFEspan model were identified.

Cues: Enablers included leadership; effective communication; organizational parity; and compatibility. Barriers included differences in organizational policies and procedures; barriers to information transfer; building an expert team; and delineation of roles.

- To what extent, if any, are these enablers/barriers still present within the LIFEspan model?

- What new successes/challenges have been encountered?

2. How does the model impact/influence a client's readiness trajectory?

- What attributes of the model (pediatric and adult) do you consider to help/hinder the preparation efforts for clients moving on to the adult health system?

- How might you alter this model to improve transition readiness?

- How willing have parents and youth been to become independent users of the healthcare system?

- Where do you see other factors/systems/service sectors fitting into the current model, if at all?

3. The LIFEspan model consists of key domains within the Growing up Ready framework.

- Which of these domains do you incorporate into your practice and how?

- Which do you consider to be the most/least influential in preparing clients for the adult healthcare system?

- Which domains do you perceive as being the most challenging for clients/clinicians to implement?

- How might you alter the framework to better meet the needs of transitioning clients?

\subsection{Data analysis}

Data analysis occurred through two main steps. First, interview transcripts were analyzed for key service delivery themes described by HCPs. Second, the themes were further analyzed, guided by a framework proposed by King and Myer [20], which conceptualizes coordinated care in relation to service integration and coordination activities.

\subsection{Interview transcripts}

Interviews were professionally transcribed verbatim and reviewed by four team members trained in qualitative methods. Summaries of all interviewee responses to the research questions were created by the RA responsible for conducting all interviews. Summaries were then reviewed by all four team members to establish the most appropriate method of coding for a thematic content analysis $[18,19]$. This type of analysis is common in qualitative research and aims to identify key elements of respondents' accounts about salient issues related to a research topic [19]. A first round of open coding of all transcripts was completed and organized using NVivo software (version 9) by one team member and verified by another. Preliminary themes regarding LIFEspan service delivery were identified and split amongst the research team members (two themes per member, no two members shared the same pair) for further, in-depth analysis and verification. Regular meetings were held to support peer debriefing. Sub-codes that were thought to best reflect service delivery themes and 'real world' experiences of LIFEspan model implementation were iden- tified, shared, and agreed upon. This method of interrater analysis supports the reliability of the coded findings [21].

\subsection{Conceptual framework analysis}

King and Meyer's [20] service integration/coordination framework was used as a lens to organize, interpret, and catalogue the service delivery themes reported by HCPs and to evaluate their contributions to coordinated care. Other conceptual models of transition, such as the direct transition, sequential transition, professional transition, and developmental transition models described by While et al. [22], were also considered; however, King and Myer's [20] framework speaks to understanding the nature of service integration and coordination functions and activities that might contribute to continuous, coordinated care for youth with disabilities and their families, specifically. In addition, this framework was chosen as it was developed based on a review of the health, social service, and rehabilitation service delivery literatures, and thus aligned with the multi-disciplinary nature of the LIFEspan model.

According to King and Meyer, the goal of coordinated care is for clients to experience services as easy to access with seamless interfaces between different services, and tailored to their needs [20]. Service coordination (i.e., activities aimed at assisting clients/families directly to locate and access services/ resources) is distinguished from service integration (i.e., activities aimed at enhancing the effectiveness of service delivery and optimizing limited resources). The scope of these activities is encompassed in three 
common approaches to delivery of coordinated care: a client/family-based service coordination approach, an agency-based service integration approach, and a system/sector-based service integration approach. Each approach consists of three main types of coordination related activities - although at varying degrees including aggregate-level service planning (i.e., designed to map out the scope and plan for service provision in a community or geographical area), administrative (i.e., designed to ensure equitable access to resources), and client-specific service delivery (i.e., designed to meet the needs of clients and families) activities. These concepts outlined in the framework were used to catalogue LIFEspan service delivery activities described by HCPs and to examine their contributions to coordinated care during the transition from the pediatric to the adult rehabilitation hospital.

\section{Results}

Following analysis of the interview data, five key themes related to LIFEspan service delivery were identified and categorized. Meaningful quotes are provided to illustrate significant themes.

\subsection{Addressing transition readiness and capacities}

The experience of healthcare transition has been well documented as an extremely stressful and challenging time for young people and their parents because they leave familiar pediatric services and clientprovider relationships [4,23,24]. Broadly, HCPs viewed LIFEspan services as positively influencing youth and family preparedness to leave pediatric services and their readiness for adult healthcare services. As one HCP observed:

So it is nice to see the comfort level of people as they go through the system going from really, really overwhelmed and "oh my god, what am I doing here?" to "you know, I can do this."

(HCP 14)

LIFEspan staff at the adult hospital acknowledged that pediatric staff had a key role in implementing transition interventions focused on building youth's skills and capacities to engage with adult service providers. As an example, pediatric staff provided youth with opportunities to practice and develop self-advocacy skills during clinic appointments. One HCP highlighted the importance of developing skills to prepare youth for navigating services in adult life:
What we're finding is that [Pediatric Hospital] has been preparing them more for [Adult Hospital].., they really come to us with a better expectation, that certain resources will no longer be available, and that they will need to do more work, and they have more sense for how to do that in order to make things happen, which is really important because we're not going to be there with them, in their homes, every day of their lives. You know, they need to develop these skills.

(HCP 6)

However, HCPs identified variances in transition readiness among youth and parents as influencing the nature and extent of targeted transition interventions. LIFEspan staff attributed the heterogeneity in transition readiness among youth to wide-ranging capacities for self-advocacy, self-management, and navigation of healthcare and community services. Such diversity is well noted within the disability literature [5, $23,25,26]$. For example, youth's readiness and capacities for self-advocacy were influenced by their preferences, health status, cognitive abilities, and family context. One HCP reflected on the various levels of support needed by youth:

Some people really don't like going to a doctor. And if they figure they can be well managed within the system and have the right connections they'll be fine with it. Some, I think, will need more than that. They'll actually need a little bit more support, either for longer or maybe a few forever, just because either the complexity or their ability to manage themselves.

(HCP 5)

As a result of varied transition readiness, interventions did not focus exclusively on youth; parents or both youth and parents were targeted. However, existing tools did not necessarily support the different interventions needed for each youth/family situation. For example, transition checklists (aimed at preparing youth for increasingly self-directed roles related to self-advocacy and self-management) were sometimes viewed by families as inappropriate for youth with severe cognitive impairments or uncertain life expectancies. Under these circumstances, parents were usually responsible for advocacy, healthcare management and transition preparation on behalf of their child. As one HCP stated:

I think there's components of that [checklist tool] ... when they've been handed that tool say, "Well, 
my kids are never moving out." Like, "This isn't happening," or, "We don't expect them to live beyond twenty years of age, so why would I make them develop this skill.

(HCP 11)

The varied youth/family circumstances and lack of appropriate tools meant staff had to be flexible and creative in incorporating both individualized and familycentred approaches to address transition readiness and capacities for each youth. These factors greatly influenced how much time staff spent on transition readiness and capacity building activities and whether youth, parents or both were the focus.

\subsection{Shifting responsibility for healthcare management from parents to youth}

One of the core aims of LIFEspan is to support a shared management model of healthcare [16,27], thus, activities related to negotiating and establishing responsibilities for managing healthcare between youth and parents were commonly reported. A shift of responsibility from parent to youth was dependent on parent readiness as well as youth transition readiness and capacities as described previously. The process was not always easy. For example, instances were noted where youth had the capacity to independently manage their healthcare but struggled to establish boundaries for parental involvement because parents were accustomed to having that responsibility. One HCP reflected on a recurring challenge when promoting a shift in responsibility for health management:

The ability ... and willingness of the youth to take those risks and then counter that with the willingness and the ability of the parents to let their youth ... their child take the risks.

(HCP 8)

HCPs had developed strategies to promote youth participation in appointments without parents, such as educating both youth and parents about the benefits of learning self-management skills and about rights to privacy, as well as providing opportunities for practice:

I try to encourage people to initiate things. So I might say, "Okay. I'll see you in six months, but if you'd like to see me before feel free to give me a call," and then write down my phone number. So I would encourage that kind of self-agency or selfmanagement.

(HCP 12)
However, for youth who did not have the capacity to manage their own healthcare (e.g., those with severe cognitive impairments), intervention activities focused on linking their families to adult-oriented services/supports and establishing legal processes for parent responsibility of healthcare (i.e., guardianship).

\subsection{Determining services in relation to organizational resources}

HCPs identified organizational resources, specifically staffing and communication, as influential factors that both supported and limited service delivery.

\subsection{Staffing}

HCPs reported that model implementation had led to the evolution of an "expert team" consisting of perspectives from multiple disciplines (see Fig. 1), as well as allowing rich, holistic collaborations among disciplines and across organizations:

I think that we actually do now have an expert team who are comfortable with the patients, and comfortable with their needs.

(HCP 7)

HCPs believed that consistency of the clinician teams through cross-appointed roles and reduced staff turnover had fostered strong relationships and rapport among LIFEspan staff. Achieving these expert transition teams was largely attributed to leadership at the mid-management level in both hospital organizations, and reflects findings from the previous study on the critical value of key decision-makers in committing staff, resources, and training time to LIFEspan services, thus, influencing changes to organizational and clinical practices [16]

Despite establishing an "expert team", HCPs reported that the design of the current service teams (e.g., predominantly part-time staff) reduced the flexibility for scheduling team meetings and for coordinating appointments and individualized intervention plans for clients. For example, because transition readiness varied among youth, different types of interventions were needed for each youth and family, which posed a challenge for efficient and consistent service delivery. HCPs believed increased time with clients and additional resources (e.g., more time for direct interventions, increased full-time equivalencies (FTEs) for staff roles, in-house programming, information/education tools, etc.) were needed to implement individualized transition and rehabilitation interventions to meet the wide-ranging needs of the growing caseloads: 
I think, for the clients, it's the amount of time that they're in the clinic appointment. I think it's the amount they can absorb, sometimes there are cognitive challenges. Every single situation is unique, so you never know what information you're providing they may follow up on or not.

(HCP 9)

I'm going to guess that there may be some sort of need ...to be an increase in maybe some of the existing resources from a ... maybe from an FTE perspective, I don't know, but ... I just know when our clinic has grown and grown over the years, that with that, we've had to grow the resources to go with it too.

(HCP 2)

The steady increase of referrals to the adult hospital over the 3 years had led to a growing caseload, without a commensurate increase in staff resources and time. As a result, staff gradually shifted towards a consultative style of service to optimize existing time resources versus providing direct interventions. As one HCP reported:

We're seeing way more patients. And, often, on a clinic day, I might get several requests and they all want to come back the next week. And I was finding, over time, that I was having to push it out a month, two months, three months, and basically do it on a, you know, priority ... well, not priority basis, really, first-come-first-serve basis.

(HCP 4)

In addition, wait times for first appointments at the adult hospital were perceived as becoming increasingly long, making the transfer seem less continuous and limiting the adult team's ability to respond to transition issues in a timely way.

\subsection{Communication}

HCPs described limitations in communicating client information between organizations, despite their beliefs that a link had been established between the pediatric and rehabilitation hospitals through crossappointed roles of staff and collaborative relationships between LIFEspan teams. In the absence of a crosshospital electronic information system, staff at the adult hospital relied on one-time paper-based summary reports sent by the pediatric team rather than full client health records readily available as needed. As a re- sult, they conducted extensive intake interviews, which were viewed as an unnecessary use of time and resources. As one HCP noted:

There's limited communication between the two teams (between the pediatric and adult hospitals) and there's no opportunity to share information because of that lack of shared network between the two.

(HCP 3)

HCPs sought guidance or leadership from decisionmakers within the organizations to address these staffing and communication challenges to support effective model delivery.

\subsection{Linking between rehabilitation services}

Most HCPs believed that a formalized link between pediatric and adult rehabilitation services had indeed been established with LIFEspan model implementation. Notably, LIFEspan staff viewed the model as successful in preparing youth and families for discharge from the pediatric rehabilitation hospital and in connecting to services at the adult rehabilitation hospital. Referring to the parent of a client, one HCP stated:

She was far better equipped [with knowledge about transfer to adult rehabilitation services] than she was thinking she might have been when her kid came to see us at $151 / 2$ and he was 18 , good to go (to adult services).

(HCP 14)

HCPs believed that model implementation strategies (e.g., cross-appointing staff to the two hospitals) supported continuity of rehabilitation care relationships. Nonetheless, HCPs identified two challenges limiting care coordination processes in this inter-disciplinary, cross-hospital model. First, HCPs suggested that processes to incorporate expanded circles of care were needed for the childhood-onset disability population:

I think that the adult side isn't used to the blatant sharing of information, whereas, in the pediatric side, the circle of care is understood to be anything that can ... any team member that's working with a client is considered to be part of that circle of care (e.g., education, community and social services), even if they're not physically in this building.

(HCP 10)

Second, legal concerns (regarding privacy of personal health information) of HCPs and both hospitals had to be clarified and addressed: 
There's been some meetings with the privacy officer ... There is still a bit of a divide in the team in terms of their comfort level with that.

(HCP 6)

Sharing client information was identified as a challenge in the previous study [16]. The results of this study further validate the need for formal communication protocols and structures (e.g., an electronic communication linkage) between the organizations.

\subsection{Linking with multi-sector services}

While connections between the partnered pediatric and adult rehabilitative LIFEspan services had been established, HCPs reported challenges in making links to other sectors, particularly community services and supports and primary care, which were considered beneficial for comprehensive and coordinated care from a broad, holistic vision of rehabilitation service delivery.

\subsection{Linking to community services and supports}

HCPs identified linkages to broader community services and supports (e.g., services related to employment, post-secondary education, intellectual disabilities and sexual health counselling) as limited, particularly in the adult rehabilitation system. One HCP stated:

I think having greater connection with the employment sector would be good, having greater connection with the transition to school sector would also be helpful, in terms of, like, college and university disability service offices.

(HCP 6)

In general, rehabilitation, education and intellectual/developmental disability services worked in silos and had not established partnerships or methods to coordinate services. HCPs indicated they worked hard to build a network of services and supports around clients to overcome poor linkages to broader community services:

I think we're supporting that ...ensuring that there's a good social network around those individuals. So part of it is ...we've developed linkages (between the youth, their families and community services).

(HCP 1)

Establishing linkages to community services and supports and building a social network around clients were considered key activities of model delivery.

\subsection{Linking to primary care}

Finding adequate support from primary care providers was also a challenge for effective service delivery. HCPs felt family doctors should be the first healthcare contacts, and should assist clients in navigating the healthcare system and in coordinating medical specialities. If a family doctor was in place, HCPs believed LIFEspan services could focus on rehabilitation and age-related goals, rather than taking on roles better aligned with primary care:

We really have a variable role in terms of primary care ...that depends on if a patient has a family doctor ... and so sometimes we have to take on primary care responsibility, and other times we don't.

(HCP 7)

We're a transition clinic. We're supposed to be dealing with issues related to preparing for discharge and entrance into adult world. And so to constantly not know where our kids are receiving their rehab medical care, their doctor care or if our nurse is supposed to be doing that is a stress for all of us.

(HCP 10)

HCPs found linking clients with primary care to be one of their greatest challenges. As one HCP stated:

One of the biggest challenges these days is ... having a primary physician who can be your contact and advocate for you within the system as well, so finding a family doctor (is vital to coordinated care).

(HCP 13)

Gaps in availability of primary care physicians are well documented and have significant implications for health outcomes of this population [16,28,29].

Overall, LIFEspan service delivery activities addressed multi-level facets of the transition process, from developing interventions focused on youth and their families to improving linkages among rehabilitation hospitals, community services and supports and primary care. These findings are consistent with the literature that conceptualizes healthcare transition as a complex issue influenced by diverse, multi-level factors (individual, family, organizational and systems factors) and emphasizes the need for multi-level interventions to address these factors $[1,13,14,23]$. 


\subsection{Service integration/coordination}

Examined through the lens of King and Meyer's [20] framework, these themes highlight the nature and scope of service delivery activities involved in the LIFEspan model implementation. Service delivery involved:

1. Client-specific service delivery activities, including interventions to improve transition readiness and capacities of youth and to establish appropriate responsibility for healthcare management between youth and parents;

2. Administrative service delivery activities, including determining services in relation to organizational resources (e.g., communication structure and staffing) and linking between rehabilitation services. However, adequacy of organizational resources at both hospitals influenced the scope, extent and focus of service delivery; and,

3. Aggregate-level service planning activities, including making links to community services and supports and to primary care, albeit these linkages were not strongly established from HCPs' perspectives.

Based on King and Myer's [20] framework, it can be argued that LIFEspan service delivery supports both client/family-based service coordination and inter-organization/agency-based service integration approaches to coordinated care.

\section{Discussion}

\subsection{Scope and nature of coordinated care service delivery}

Thematic content analysis of the interview transcripts identified five main service delivery activities: (i) addressing transition readiness and capacities; (ii) shifting responsibility for healthcare management from parents to youth; (iii) determining services in relation to organizational resources (e.g., staffing and communications); (iv) linking between rehabilitation services; and, (v) linking to multi-sector services, including employment, education, community and social services and supports, and to primary care. The nature and scope of these activities contributed to client/family-based service coordination and inter-organization/agency-based service integration approaches to coordinated care. These findings validate the intent of LIFEspan service delivery activ- ities - that is, to promote continuous and coordinated transitional care from one rehabilitation hospital to another. In this regard, HCPs believed a linked model of continuous and coordinated care between pediatric and adult rehabilitation services had been established based on current service delivery. However, making linkages to primary care and services that addressed broader health-related domains (e.g., post-secondary education, employment, housing, personal care and income supports) was also viewed as an important component of comprehensive rehabilitative practices that promote participation and engagement in community life.

From the lens of King and Myer's [20] framework, it appears that deficits in aggregate-level service planning activities, for example, limited collaboration and service integration with other sectors (i.e., primary care, education and community and social services), impeded comprehensive service coordination for this population during the transition to adult services. The lack of a systems/sector-based service integration approach resulted in many of the challenges experienced by HCPs in providing comprehensive, transitional rehabilitative care. Without broader sector partnerships, care coordination may be limited to the rehabilitation sector within the current model. Table 2 provides several recommendations to enhance and augment current service delivery activities to support broader service integration of rehabilitation with other sectors.

HCPs also provided insight into factors influencing their practical experiences of model implementation. These findings will be discussed further in relation to the LIFEspan model itself, and to the transition literature.

\subsection{Successes and challenges of service delivery}

HCPs described factors, both successes and challenges, influencing their experiences of 'real world' implementation across three phases of the LIFEspan model. In relation to Phases 1 (transition preparation in pediatric services) and 2 (transfer services), they believed service delivery activities had prepared youth and families for the transfer to adult rehabilitation services and had indeed established a link between the pediatric and adult hospitals. In particular, HCPs believed that an 'expert' multi-disciplinary transition team had evolved, which supported continuity of care through cross-appointments of staff and low staff turnover. Small integrated teams with staff continuity have been shown to support continuity of care [30]. Consistency 
Table 2

Recommendations for a systems/sector-based service coordination approach

\begin{tabular}{ll}
\hline $\begin{array}{l}\text { Activities of care } \\
\text { coordination }{ }^{20}\end{array}$ & Recommendations \\
\hline $\begin{array}{l}\text { Service planning } \\
\text { activities }\end{array}$ & $\begin{array}{l}\text { - Establish partnerships with community resources (from rehabilitation, healthcare, education and employment sectors) } \\
\text { to support coordinated care } \\
-\end{array}$ \\
& - Establish partnerships with primary care resources to support model sustainability \\
& - Increase staff working hours, full-time and cross-appointed positions where finances allow \\
Administrative & - Clarify service delivery expectations for staff and create clear guidelines to assist staff in managing growing caseloads \\
activities & - Review service delivery and supporting processes with a focus on increasing efficiency and maximizing client-provider \\
& interactions \\
& - Create a cross-hospital shared information network \\
& - Promote youth self-advocacy through knowledge and skill building opportunities as appropriate \\
Client specific & Encourage family support of youth self-management of healthcare through early introduction of transition information \\
activities & and opportunities to practice skills \\
& - Develop and adapt care to meet individualized needs and goals of youth and their families
\end{tabular}

of staff resulted in improved communication and collaboration in the transfer of care between hospitals and better transition preparation and service transfers for youth and families. Close collaboration and communication between families and organizations are considered key determinants of the quality of transitional rehabilitation planning for adolescents with CP [31,32].

The heterogeneous nature of $\mathrm{CP}$ and $\mathrm{ABI}$ was a challenge for transition preparation (Phase 1). Both diagnoses present with varied combinations of impairments, and activity and participation limitations [31]. As a result, youth and families had diverse rehabilitation needs, as well as various degrees of transition readiness and capacities, which influenced the scope and extent of transition interventions and the amount of time needed to plan and implement these interventions. HCPs strived to incorporate both client- and familycentered practices in clinic, which also increased the required staff and time resources. The desire for more time to develop and implement individualized intervention plans is not unusual in itself. The literature supports transition interventions at the individual level that emphasize developing skills and knowledge for self- or shared-management of health [30-35] and the provision of family support [26], further validating the need for LIFEspan activities aimed at both youth and their families. However, in relation to LIFEspan services, the complement of part-time HCPs between the two sites meant less flexibility for scheduling time to meet and to collaborate on intervention plans, and thus, less continuity of care. Sufficient staff time and appropriate clinic structures have been highlighted as vital for implementing organizational change to support successful transition program implementation [36].

For transfer of services (Phase 2), the successful transfers of youth to the adult hospital meant in- creased caseloads without commensurate increases in staff time dedicated to adult LIFEspan services. Wait times for first appointments at the adult hospital increased over time, which HCPs believed made the transfer to adult services appear less coordinated and continuous than intended. A lesson learned is that organizations should regularly evaluate service delivery outcomes and be prepared to adjust services to growing caseloads. It can be argued that the LIFEspan team will become increasingly efficient in service delivery as they gain more experience; regardless, the caseload will reach a certain point when the staff time allocation will only allow for minimal client-provider interactions and likely have detrimental effects on service delivery. Organizations need to plan for the long-term success and sustainability of transition programs [36]. Potential methods for improving efficiencies in service delivery were suggested in this study, which are also supported in the literature, including a shared electronic health record network [16], clarification of laws related to privacy of health information $[28,36]$, and processes for expanding circles of care beyond the youth, parents and LIFEspan team [15].

In adult services (Phase 3), HCPs believed that continuity of rehabilitative care had been achieved through LIFEspan service delivery. As discussed in the previous section, service delivery activities did not appear to support strong linkages to primary care, education, and community services and supports. Although the LIFEspan model continued to address the traditional hurdles associated with healthcare transitions, staff struggled to make connections to services and supports that could promote positive outcomes associated with social determinants of health (e.g., employment, housing, income, etc.). With respect to primary 
care, many youth did not have access to a family doctor. LIFEspan staff believed they were taking on some roles and activities normally conducted by family doctors. Addressing primary care needs meant less time for the team to focus on transitional rehabilitative goals and interventions. Although changes to organizational processes and HCPs' practices had improved the process of transfer to adult rehabilitative care, service delivery may not address the complexity and diverse factors associated with the transition to adult life [14]. The need for multi-sector coordination at the systems level, to address existing gaps among health, education, employment and social service sectors, has been well documented in the literature $[10,26,37,38]$. Table 2 provides recommendations for LIFEspan service delivery activities to improve multi-sector service coordination.

\subsection{Broader transition literature}

Healthcare transition has been described as a complex phenomenon influenced by multiple, diverse factors $[4,23,39,40]$, including skills and knowledge of self- or shared-management of health at the individual level [33-35], family support at the interpersonal level [26], co-ordination between pediatric and adult services at the organizational level [41,42] and resources to support the transition to adulthood at the policy level $[4,10,21]$. LIFEspan service delivery incorporated many activities that addressed multi-level influences on healthcare transitions, including interventions for both youth and parents to improve transition readiness and capacities and to promote a shift in responsibility for health management, and formalized processes to support the continuity of care between hospitals. From the HCPs' perspectives in this study, these activities contributed to care coordination and linkages between pediatric and rehabilitation services; however, making linkages to services and supports outside of the rehabilitation sector remained a challenge.

Youth with disabilities have complex, diverse healthcare needs that go beyond medical needs [23]. They experience poor health outcomes such as decreased health status [25], but also poor outcomes in healthrelated domains, such as participation in education, employment, relationships and community activities [3]. This complex array of needs requires involvement and coordination of multiple service systems, including health, education and social services and a broad view of health beyond medical health [7,12]. This study found that poor connections to education, employment and intellectual disability services and supports, as well as to primary care continued to be a challenge, even though the LIFEspan model implementation had addressed the gap in transition to adult rehabilitative care. It was difficult for LIFEspan staff to find appropriate services for youth and families in the community. In general, multi-sector services fell into policy silos that were not well connected or coordinated. Leadership at the public policy level is needed to improve multi-sector service coordination and integration for youth in transition to adult services $[14,40]$.

\subsection{Methodological considerations}

In discussing the limitations of these findings, bias may be present as HCPs reflected on a single and very specific partnership service. In addition, the length and depth of interviews varied and involved both in-person and telephone interviews and thus, may not be representative of LIFEspan HCPs' perspectives as a whole. However, all invited eligible staff did participate in an interview (100\% response rate), thus, it can be argued that the findings are representative of a collective, multi-disciplinary perspective. There is a risk that findings may only be applicable to implementing transition models in rehabilitation and in relation to youth with $\mathrm{CP}$ and $\mathrm{ABI}$ and/or to this particular healthcare setting. However, the aim was not statistical generalization, but rather to understand general themes related to delivery of coordinated care that might be important to consider in other settings and for similar populations. Although the theme about addressing transition readiness and capacity is likely related in part to neurological aspects of $\mathrm{CP}$ or $\mathrm{ABI}$ diagnoses, the other themes are considered generic and thus, relevant to other pediatric to adult service transitions. Transferability of findings to other populations is supported by the emphasis placed on administrative and aggregate-level service planning activities, rather than client-specific activities reflected in the interview accounts. These findings highlight service delivery activities and factors influencing their implementation that can inform transitional care and service delivery in other healthcare settings and for other client populations.

\section{Conclusion}

LIFEspan service delivery demonstrated a multilevel approach to transitional rehabilitative care with interventions focused predominantly on change at 
client/family and organization/inter-organization levels. Service delivery activities contributed to rehabilitation service coordination for youth and families and to service integration between two hospitals. However, without interventions that address service integration across policy sectors, service coordination for youth will be limited to rehabilitation services under the LIFEspan model. Cross-sector partnerships (e.g., primary care, education and employment services and services for individuals with intellectual disabilities) at the systems level are required to optimize continuity of comprehensive coordinated care. These findings provide contextual information about implementing healthcare transition best practices through the LIFEspan model and identify changes needed to support continuous, coordinated rehabilitative care for youth with $\mathrm{CP}$ and $\mathrm{ABI}$. Further work might examine the scope and context of rehabilitation practice in relation to general transition principles developed for the broad healthcare sector. These recommendations and continued evaluation through the LETS study can inform evolution and sustainability of the LIFEspan model.

\section{Acknowledgements}

Funding for the LETS Study was provided by the Ontario Neurotrauma Foundation (2008-ABILSMODEL-706). The authors would like to thank the HCPs who provided valuable insights into LIFEspan model implementation. We also thank the Toronto Central LHIN for funding the LIFEspan Service. We would like to acknowledge Irina Tsybina and Ashley Lacombe-Duncan for their assistance in LETS study coordination, and Patricia McKeever and Helen Healy for their assistance in securing funding.

\section{Clinical trial registration}

The LETS study is registered as a clinical trial: http://www.clinicaltrials.gov, ID NCT00975338.

\section{Conflict of interest}

The authors report no conflict of interest.

\section{References}

[1] D. Wood, J. Reiss, M. Ferris, L. Edwards and J. Merrick, Transition from pediatric to adult care, Int J Child Adolesc Health 3(4) (2011), 445-447.
[2] S.M. Sawyer, S. Drew, M.S. Yeo and M.T. Britto, Adolescents with a chronic condition: challenges living, challenges treating, Lancet 369(9571) (2007), 1481-1489.

[3] D. Stewart, C. Stavness, G. King, B. Antle and M. Law, A critical appraisal of literature reviews about the transition to adulthood for youth with disabilities, Phys Occup Ther Pediatr 26(4) (2006), 5-24.

[4] J. Reiss and R. Gibson, Health care transition: destinations unknown, Pediatrics 110(6 Pt 2) (2002), 1307-1314.

[5] S.T. Callahan and W.O. Cooper, Access to health care for young adults with disabling chronic conditions, Arch Pediatr Adolesc Med 160(2) (2006), 178-182.

[6] L. Fiorentino, D. Datta, S. Gentle, D.M. Hall, V. Harpin, D. Phillips et al., Transition from school to adult life for physically disabled young people, Arch Dis Child 79(4) (1998), 306-311.

[7] J.E. McDonagh, Child-adult interface. The adolescent challenge, Nephrol Dial Transplant 15(11) (2000), 1761-1765.

[8] S. Ried, Transition of Youth From Pediatric to Adult Care: Physician's Perspective and Recommendations, Top Spinal Cord Inj Rehabil 16(1) (2010), 38-47.

[9] C.J. Stevenson, P.O. Pharoah and R. Stevenson, Cerebral palsy - the transition from youth to adulthood, Dev Med Child Neurol 39(5) (1997), 336-342.

[10] R.M. Viner, Transition of care from paediatric to adult services: one part of improved health services for adolescents, Arch Dis Child 93(2) (2008), 160-163.

[11] D. Stewart, M. Law, N. Young, H. Healy, M. Forhan, J. Burkey-Gaffney et al., Understanding the transitional tensions of youth with disabilities in Canada: Identifying key research gaps. Penultimate report to Human Resources Social Development Canada (HRSDC). Hamilton, ON; McMaster University, 2009.

[12] C. Betz and K. Smith, Measuring health care transition planning outcomes: Challenges and issues, Int J Child Adolesc Health 3(4) (2011), 561-566.

[13] J.W. Gorter, D. Stewart and M. Woodbury-Smith, Youth in transition: care, health and development, Child Care Health Dev 37(6) (2011), 757-763.

[14] Y. Hamdani, A. Jetha and C. Norman, Systems thinking perspectives applied to healthcare transition for youth with disabilities: A paradigm shift for practice, policy and research, Child Care Health Dev 37(6) (2011), 806-814.

[15] S. Shanske, J. Arnold, M. Carvalho and J. Rein, Social Workers as Transition Brokers: Facilitating the Transition From Pediatric to Adult Medical Care, Soc Work Health Care 51(4) (2012), 279-295.

[16] S. Kingsnorth, S. Lindsay, J. Maxwell, I. Tsybina, H. Seo, C. Macarthur et al., Implementation of the LIFE span model of transition care for youth with childhood onset disabilities, Int J Child Adolesc Health 3 (2011), 547-561.

[17] I. Tsybina, S. Kingsnorth, J. Maxwell, M. Bayley, S. Lindsay, P. McKeever et al., Longitudinal Evaluation of Transition Services ("LETS Study"): protocol for outcome evaluation, $B M C$ Pediatr 12 (2012), 51.

[18] M.Q. Patton, Qualitative evaluation and research methods 2nd ed. Thousand Oaks, CA: Sage Publications, 1990.

[19] J. Green and H. Thorogood, Qualitative Methodology and Health Research. In: Qualitative Methods for Health Research. London: Sage Publications, 2004, pp. 3-26.

[20] G. King and K. Meyer, Service integration and co-ordination: A framework of approaches for the delivery of co-ordinated care to children with disabilities and their families, Child Care Health Dev 32(4) (2006), 477-492. 
[21] C. Pope, S. Ziebland and N. Mays, Qualitative research in health care, Analysing qualitative data, BMJ 320(7227) (2000), 114-116

[22] A. While, A. Forbes, R. Ullman, S. Lewis, L. Mathes and P. Griffiths, Good practices that address continuity during transition from child to adult care: synthesis of the evidence, Child Care Health Dev 30(5) (2004), 439-452.

[23] J.A. Binks, W.S. Barden, T.A. Burke and N.L. Young, What do we really know about the transition to adult-centered health care? A focus on cerebral palsy and spina bifida, Arch Phys Med Rehabil 88(8) (2007), 1064-1073.

[24] P. Scal and M. Ireland, Addressing transition to adult health care for adolescents with special health care needs, Pediatrics 115(6) (2005), 1607-1612. Epub 2005/06/03.

[25] N.L. Young, W.S. Barden, W.A. Mills, T.A. Burke, M. Law and K. Boydell, Transition to adult-oriented health care: perspectives of youth and adults with complex physical disabilities, Phys Occup Ther Pediatr 29(4) (2009), 345-361.

[26] J.G. Reiss, R.W. Gibson and J.R. Walker, Health care transition: youth, family, and provider perspectives, Pediatrics 115(1) (2005), 112-120.

[27] G.M. Kieckhefer and C.M. Trahms, Supporting development of children with chronic conditions: from compliance toward shared management, Pediatr Nurs 26(4) (2000), 354-363.

[28] H. Gleeson, S. McCartney and V. Lidstone, 'Everybody's business': transition and the role of adult physicians, Clin Med 12(6) (2012), 561-566

[29] L. Pickler, Y. Kellar-Guenther and E. Goldson, Barriers to transition to adult care for youth with intellectual disabilties, Int J Child Adolesc Health 3(4) (2011), 575-584.

[30] H. Gleeson and G. Turner, Transition to adult services, Archives of Disease in Childhood Education and Practice Edition 97(3) (2012), 86-92. Epub 2011/10/08

[31] I. Jeglinsky, A.L. Salminen, E.B. Carlberg and I. Autti-Ramo, Rehabilitation planning for children and adolescents with cerebral palsy, J Pediatr Rehabil Med 5(3) (2012), 203-215.

[32] M.C. Podvey, J. Hinojosa and K. Koenig, The transition experience to pre-school for six families with children with disabilities, Occup Ther Int 17(4) (2010), 177-187.

[33] C. Gall, S. Kingsnorth and H. Healy, Growing up ready: A shared management approach, Phys Occup Ther Pediatr 26(4) (2006), 47-62.

[34] A. Kennedy, F. Sloman, J. Douglass and S. Sawyer, Young people with chronic illness: The approach to transition, Intern Med J 37(8) (2007), 555-560.

[35] A.W. Schultz and G.S. Liptak, Helping adolescents who have disabilities negotiate transitions to adulthood, Issues Compr Pediatr Nurs 21(4) (1998), 187-201.

36] C. Price, S. Corbett and G. Davey-Pearce, Barriers and facilitators to implementing a transition pathway for adolescents with diabetes: A health professional's perspective, Int J Child Adolesc Health 3(4) (2011), 489-498.

[37] G.A. King, P.J. Baldwin, M. Currie and J. Evans, The Effectiveness of Transition Strategies for Youth With Disabilities, Child Health Care 35(2) (2006), 155-178.

[38] W.M. McDonnell, I. Kocolas, G.E. Roosevelt and A.T. Yetman, Pediatric emergency department use by adults with chronic pediatric disorders, Arch Pediatr Adolesc Med 164(6) (2010), 572-576.

[39] P. Rapley and P.M. Davidson, Enough of the problem: A review of time for health care transition solutions for young adults with a chronic illness, J Clin Nurs 19(3-4) (2010), 313323.

[40] G. Wang, B.B. McGrath and C. Watts, Health care transitions among youth with disabilities or special health care needs: An ecological approach, J Pediatr Nurs 25 (2010), 505-550.

[41] N.L. Young, The transition to adulthood for children with cerebral palsy: what do we know about their health care needs? J Pediatr Orthop 27(4) (2007), 476-479.

[42] J.E. McDonagh, T.R. Southwood, K.L. Shaw and G. Bristish, Paediatric Rheumatology, Unmet education and training needs of rheumatology health professionals in adolescent health and transitional care, Rheumatology 43(6) (2004), 737743 . 